\title{
Comunicação
}

[Communication]

\section{Efeitos do trimetilaminoetano (TES) e ringer lactato em sêmen de macacos-aranha mantidos em cativeiro (Ateles paniscus e A. marginatus)}

\author{
[Effects of TES and a ringer-lactato diluent in semen from captive spider monkeys \\ (Ateles paniscus e A. marginatus)]
}

\author{
K.S.M. Silva ${ }^{1}$, H.F.L. Ribeiro ${ }^{2}$, R. R. Valle $^{3}$, J.S. Sousa ${ }^{4}$, A.O. Silva ${ }^{4}$, E. M. Barbosa ${ }^{5}$ \\ ${ }^{1}$ Instituto Evandro Chagas/Centro Nacional de Primatas Ananindeua - Belém, PA \\ ${ }^{2}$ Instituto de Saúde e Produção Animal -Universidade Federal Rural da Amazônia - Belém, PA \\ ${ }^{3}$ Faculdade de Medicina Veterinária e Zootecnia - Universidade de São Paulo - São Paulo, SP \\ ${ }^{4}$ Central de Biotecnologia e Reprodução Animal - Universidade Federal do Pará - Belém, PA \\ ${ }^{5}$ Aluno de pós-graduação - Universidade Federal do Pará - Belém, PA
}

A biotecnologia aplicada à reprodução é uma ferramenta importante para a conservação de espécies ameaçadas de extinção (Wildt et al., 1993). Algumas espécies de primatas não humanos neotropicais têm sido amplamente agredidas por ações antrópicas, em especial as espécies pertencentes ao gênero Ateles, popularmente chamados de macaco-aranha. Ateles marginatus, por exemplo, encontram-se na lista vermelha de animais ameaçados da International Union for Conservation of Nature and Natural Resources, desde 2000 (IUCN, 2012). Poucos estudos são realizados acerca do status populacional dessas espécies e, menos ainda, sobre os dados reprodutivos e a reprodução assistida. Considerando também o custo elevado de alguns equipamentos e materiais necessários à conservação dos gametas e embriões, como os diluentes, há um entrave à aplicação eficiente de programas de reprodução assistida voltados a essas espécies. O custo do TES, por exemplo, é oito vezes maior que o custo do diluente à base de Ringer Lactato. Desta forma, o presente estudo objetivou o estudo comparativo dos diluentes TES e CEBRAN II, à base de ringer lactato, como criopreservadores de sêmen dessas espécies, no intuito de somar para o sucesso na criopreservação e auxiliar a diminuição dos custos de projetos ligados à biotecnologia aplicada à conservação de animais em situação de risco.

Recebido em 14 de novembro de 2011

Aceito em 29 de novembro de 2012

E-mail: klenasilva@iec.pa.gov.br
Por meio de eletroejaculação, foram colhidos ejaculados obtidos de dois exemplares de Ateles paniscus e um exemplar de Ateles marginatus, machos e adultos, mantidos em cativeiro no Centro Nacional de Primatas (Cenp/IEC/SVS/MS). Foi utilizado protocolo sequencial de 90 estímulos divididos em três séries de 30 estímulos variando de 2 a 6 volts. Os animais foram submetidos a exame clínicoandrológico e biometria testicular, antes da colheita seminal. Após mensuração do volume, os ejaculados foram imediatamente analisados quanto à cor, ao aspecto e ao $\mathrm{pH}$ do conteúdo, utilizando-se fita reativa para $\mathrm{pH}$, para descartar a presença de urina na amostra. O TES e o CEBRAN II foram preparados na Central de Biotecnologia Reprodutiva Animal da Universidade Federal do Pará (Cebran/UFPA), conservados em freezer e descongelados a cada dia de colheita, 30 minutos antes, em água à temperatura ambiente. Para assegurar melhor qualidade dos diluentes, esses tiveram $\mathrm{pH}$ ajustados para valor 8.0 no momento da preparação da solução-mãe, de forma a aproximar-se do $\mathrm{pH}$ do sêmen dos animais. Foram, então, adicionados $20 \mu \mathrm{L}$ de cada ejaculado obtido a cada colheita à mesma quantidade dos diluentes TES e CEBRAN II, em lâmina pré-aquecida a $37^{\circ} \mathrm{C}$, para avaliação de motilidade, vigor e do tempo de resistência dos espermatozoides nos diluentes. Ao mesmo tempo, outros $20 \mu \mathrm{L}$ do sêmen fresco eram 
colocados também em lâmina pré-aquecida a $37^{\circ} \mathrm{C}$ para avaliar motilidade, vigor e tempo de resistência dos espermatozoides em condições externas. Após a colheita, os ejaculados com maiores volumes foram diluídos, em proporção de 2:1, ainda nos próprios tubos coletores, tanto com o diluente TES quanto com o CEBRAN II. Os tubos foram levados à refrigeração durante uma hora, para que fosse possível não parar a colheita para obtenção de novos ejaculados no mesmo animal. Após essa primeira hora de refrigeração, o produto da diluição foi envasado em minitubos (capacidade $0,25 \mathrm{~mL}$ ), e estes foram vedados com massa de modelar atóxica, sendo devidamente identificados com informações sobre a tatuagem do animal, o diluente utilizado e a data de envase. Em seguida, os minitubos foram levados ao processo de congelamento com o seguinte protocolo utilizado pelo Cebran: refrigeração a $4^{\circ} \mathrm{C}$; congelamento em freezer $\left(-4^{\circ} \mathrm{C}\right)$ por duas horas; 20 minutos em imersão em vapor de nitrogênio líquido $\left(-60^{\circ} \mathrm{C}\right)$ em uma caixa de isopor com capacidade de 30 litros, contendo grade de aço inoxidável suspensa a $10 \mathrm{~cm}$ do nível de nitrogênio líquido; imersão dos minitubos em botijão contendo nitrogênio líquido $\left(-196^{\circ} \mathrm{C}\right)$.

Para descongelamento, os minitubos foram imersos em banho-maria a $37^{\circ} \mathrm{C}$ por cinco minutos, e, posteriormente, uma gota de cada dose foi avaliada quanto à motilidade $(\%)$ e ao vigor, considerando-se uma escala de um a cinco, mediante o uso de microscópio de contraste de fase, correlacionando-se o meio criopreservador com o sêmen avaliado. Novas avaliações de motilidade e vigor foram observadas após meia-hora.

A média de volume de ejaculado obtido foi de $1,94 \mathrm{~mL}( \pm 0,83)$ e a média de concentração espermática obtida foi de $3.020 .000 \mathrm{sptz} / \mathrm{mL}$ $( \pm 275,97)$. O pH 8 foi observado em todas as amostras e todos os exemplares apresentaram coagulação seminal.

Como somente três amostras foram utilizadas para descrever os resultados obtidos, não foi possível realizar análise estatística. A análise descritiva das avaliações pode ser observada na Tab. 1, em que o momento 0 (zero) refere-se ao momento de análise logo após a ejaculação.

Os resultados do teste de termorresistência (TTR) demonstraram que as amostras com sêmen criopreservado exibiram espermatozoides vivos e apresentando motilidade e vigor.

Tabela 1. Resultado da avaliação de motilidade e vigor do sêmen a fresco e diluído dos exemplares de Ateles

\begin{tabular}{|c|c|c|c|c|c|c|c|}
\hline \multirow[t]{2}{*}{ Animais } & \multirow{2}{*}{$\begin{array}{l}\text { Tempo de } \\
\text { avaliação }\end{array}$} & \multicolumn{2}{|c|}{ Sptz in natura } & \multicolumn{2}{|c|}{ Com TES } & \multicolumn{2}{|c|}{ Com Ringer } \\
\hline & & Motilidade & Vigor & Motilidade & Vigor & Motilidade & Vigor \\
\hline \multirow[t]{3}{*}{$\begin{array}{l}\text { AK-AAD } \\
\text { (Ateles } \\
\text { marginatus) }\end{array}$} & $\begin{array}{l}\text { Momento } 0 \\
\text { (zero) }\end{array}$ & $60 \%$ & 3 & $60 \%$ & 3 & $60 \%$ & 3 \\
\hline & $\begin{array}{l}\text { Após } 30 \\
\text { minutos }\end{array}$ & - & - & $40 \%$ & 2 & $10 \%$ & 1 \\
\hline & $\begin{array}{c}\text { Após } 3 \\
\text { horas }\end{array}$ & - & - & $30 \% *$ & 2 & - & - \\
\hline \multirow{2}{*}{$\begin{array}{l}\text { AI-AAB } \\
\text { (Ateles } \\
\text { paniscus) }\end{array}$} & $\begin{array}{l}\text { Momento } 0 \\
\text { (zero) }\end{array}$ & $20 \%$ & 1 & - & - & - & - \\
\hline & $\begin{array}{l}\text { Após } 30 \\
\text { minutos }\end{array}$ & - & - & - & - & - & - \\
\hline \multirow{2}{*}{$\begin{array}{l}\text { AI-AAD } \\
\text { (Ateles } \\
\text { paniscus) }\end{array}$} & $\begin{array}{l}\text { Momento } 0 \\
\text { (zero) }\end{array}$ & $30 \%$ & 2 & $10 \%$ & 1 & - & - \\
\hline & $\begin{array}{l}\text { Após } 30 \\
\text { minutos }\end{array}$ & - & - & $10 \%$ & 1 & - & - \\
\hline
\end{tabular}

*Os espermatozoides avaliados nesta amostra com TES foram observados mortos 12 horas após a colheita. As lâminas foram mantidas em condições de temperatura ambiente $\left(28^{\circ} \mathrm{C}\right)$. 
Diluentes à base de TES já demonstraram boa utilização na criopreservação de sêmen de outras espécies, como Chlorocebus aethiops (Seier et al., 1993) e Macaca fascicularis (Sankai et al., 1994; Tollnert et al., 1990; Valle et al., 2004; Li et al, 2005).

Sabe-se que fatores como diferenças individuais, estação, método de colheita, componentes do diluente, $\mathrm{pH}$ do diluente e escolha do crioprotetor têm enorme influência sobre a viabilidade de espermatozoides pós-congelamento (Agca et al., 2005). $\mathrm{O}$ pH do diluente variando entre 7,2 e 8,0 leva a um melhor desempenho de motilidade dos espermatozoides após descongelamento (Mahone e Dukelow, 1978).

A porcentagem de glicerol presente nos diluentes também pode influenciar. A porcentagem desse crioprotetor, presente tanto no diluente CEBRAN II quanto no diluente TES utilizados neste experimento, foi de 7\%. Si e colaboradores (2004) observaram que diluentes contendo $5 \%$ de glicerol mostraram-se mais eficientes em sêmen de Macaca mulatta, enquanto Tollnert e sua equipe (1990) observaram melhor perfomance desse crioprotetor em concentração a $3 \%$ em sêmen de Macaca fascicularis.

Sendo ringer lactato uma solução à base de sais minerais, essa composição pode ter influenciado nos resultados observados, pois Li e colaboradores (2005) relatam que, no congelamento do sêmen de bovinos, ratos e macaco Rhesus, a motilidade caiu drasticamente no pós-descongelamento devido à exposição a íons de sal.

O diluente TES é atualmente utilizado para diversas espécies de mamíferos, como bovinos e bubalinos. Segundo a Cebran/UFPA, o TES apresenta um custo de, aproximadamente, 25 dólares por litro, contra um valor de três dólares por litro do diluente CEBRAN II. A solução de ringer lactato já foi comprovada como bom diluidor de sêmen de búfalos no experimento de Silva e pesquisadores parceiros (2002). Neste experimento, o ringer lactato se mostrou tão eficiente quanto o TES para manutenção da motilidade e do vigor dos espermatozoides de bubalinos após descongelamento, ambos apresentando índices similares. Ribeiro (1991) também encontrou um alto índice de eficiência $(53,3 \%)$ do ringer lactato como diluente para sêmen de bubalinos, no uso a campo, porém esse diluente ainda não havia sido testado em sêmen de espécies de Ateles, somente para primatas Alouatta caraya, em experimento realizado sem finalidade de criopreservação, em que se mostrou significativamente diferente para motilidade em relação ao diluente TES $(74 \pm 20 \%$ e $71 \pm 20 \%$, respectivamente) (Valle, 2004). Ao se observar o resultado da avaliação de motilidade e vigor do sêmen a fresco e diluído e pós-descongelamento, apenas pode-se sugerir que o diluente TES demonstra-se mais eficiente para viabilização de espermatozoides de Ateles, pois o número de amostras foi insuficiente para realizar qualquer afirmação sobre a eficiência dos diluentes propostos. No entanto, mais estudos são necessários. Alterações na composição dos diluentes são recomendadas, de modo a otimizar não só a osmolaridade e o pH necessários para preservação do sêmen desses primatas, mas também a concentração de crioprotetor mais próxima do ideal, assim como os antibióticos e açúcares utilizados e suas concentrações.

Palavras-chave: sêmen, diluentes, Ateles, cativeiro

\begin{abstract}
The performances of the diluents TES and CEBRAN II were compared as cryopreservatives of semen from non human primates of the genus Ateles. The experiment was carried out using one Ateles marginatus and two Ateles paniscus specimens, males and adults, maintained in the same captivity conditions at the National Center of Primates (CENP-SVS/MS). The animals were subjected to clinical and andrological examinations - testicular biometry - before the semen collection by eletroejaculation. Evaluations of motility and forward movement in the fresh semen were made. Semen were made dilution was made with the diluents TES and CEBRAN II. The ejaculates were diluted with the diluents (2:1proportion), packed in $0.25 \mathrm{~mL}$ plastic straws and cryopreserved in liquid nitrogen. After thawing, the packed ejaculates were appraised in thermo resistance test (TTR). The averages of volume and concentration were, respectively, $1.94 \mathrm{~mL}(0.83)$ and 3,020,000 sptz/mL (275.97). The pH 8 and seminal
\end{abstract}


coagulation were observed in all samples. The results suggest that the TES diluent presents better efficiency in the preservation of Ateles semen than CEBRAN II.

Keywords: semen, diluents, Ateles, captivity

\section{REFERÊNCIAS}

AGCA, Y.; LIU, J; MULLEN, S. et al. Chimpanzee (Pan troglodytes) spermatozoa osmotic tolerance and cryoprotectant permeability characteristics. J. Androl., v.26, p.470-477, 2005.

IUCN 2012. The IUCN Red List of Threatened Species. Version 2012.1. Disponível em: <http://www.iucnredlist.org>. Acessado em: 19 jun. 2012.

LI, Y.; CAI, K.; KOVACS, A. et al. Effects of various extenders and permeating cryoprotectants on cryopreservation of cynomolgus monkey (Macaca fascicularis) spermatozoa. J. Androl., v.26, p.387-395, 2005.

MAHONE, J.P.; DUKELOW, W.R. Semen preservation in Macaca fascicularis. Lab. Anim. Scie., v.28, p.556-561, 1978.

RIBEIRO, H.F.L.; SILVA, A.O.A.; SOUSA, J.S. et al. The use of thermoresistance test for water buffalo semen (preliminary report). In: WORLD VETERINARY CONGRESS, 1991, Rio de Janeiro. Proceedings... Rio de Janeiro: [s.n.] 1991. (Abstract).

SANKAI, T.; TERAO, K.; Yanagimachi, R. et al. Cryopreservation of spermatozoa from Cynomolgus Monkeys (Macaca fascicularis). J. Reprod. Fertil., v.101, p.273-278, 1994.
SEIER, J.V.; CONRADIE, E.; OETTLE, E.E. et al. Cryopreservation of vervet monkey semen and recovery of progressively motile spermatozoa. J. Med. Primatol., v.22, p.355-359, 1993.

SI, W.; ZHENG, P.; LI, Y. et al. Effect of glycerol and dimethyl sulfoxide on cryopreservation of rhesus monkey (Macaca mulatta) sperm. Amer. J. Primatol., v.62, p.301-306, 2004.

SILVA, A.O.A.; MOTA, A.V.; RIBEIRO, H.F.L. et al. Preliminar report on ringer-lactate solution as an alternative diluter for bufallo semen. In: BUFALLO SYMPOSIUM OF AMERICAS, 2002, Belém. Proceedings... Belém: [s.n.] 2002. p.467-70. (Abstract)

TOLLNERT, T.L.; VANDEVOORT, C.A.; OVERSTREET, J.W. et al. Cryopreservation of spermatozoa from cynomolgus monkeys (Macaca fascicularis). J. Reprod. Fertil., v.90, p.347-352, 1990.

VALLE, R.R.; GUIMARÃES, M.A.B.V.; MUNIZ, J.A.P.C. et al. Collection and evaluation of semen from captive howler monkeys (Alouatta caraya). Theriogenology, v.62, p.131-138, 2004.

WILDT, D.E.; SEAL, U.S.; RALL, W.F. Genetic resource banks and reproductive technology for wildlife conservation. In: CLOUD, J.G. Genetic Conservation of Salmonid Fishers. New York: Thorgaard Plenum Press, 1993. p.159-173. 